the proximal interphalangeal (PIP) $(0.26)$ and moderate at wrist $(0,56)$, ultrasound detected more erosions than standard radiography $(56 \%$ versus $17 \%$ ), and a statistically significant correlation was observed between the onset of RA, its stage, and the ultrasound score.

Conclusion: Evaluation of a heterogeneous group demonstrated that ultrasound completes clinical examination information, particularly at the PIP and MCP levels, and detects erosions earlier than standard imaging.

Disclosure of Interests: None declared

DOI: 10.1136/annrheumdis-2019-eular.5195

\section{THU0608 $\quad$ CAPILLAROSCOPIC DIFFERENCES IN PRIMARY BILIARY CHOLANGITIS WITH OR WITHOUT SCLERODERMA AND RAYNAUD'S PHENOMENON, A PRELIMINARY STUDY}

Mayka Freire $^{1}$, Sandra Martínez ${ }^{2}$, Bernardo Sopeña ${ }^{1,2}$, lago Carballo ${ }^{1}$, Esteban Otero ${ }^{1}$, Nestor Vazquez-Agra ${ }^{1}$, Ana-Teresa Marques ${ }^{1}$, Arturo GonzálezQuintela ${ }^{1,2}$. ${ }^{1}$ Hospital Clínico Universitario de Santiago, Santiago de Compostela, Spain; ${ }^{2}$ Universidad de Santiago de Compostela, Santiago de Compostela, Spain

Background: A high proportion of capillaroscopic alterations have been reported in patients with Primary Biliary Cholangitis (PBC) (1). Association with an abnormal capillaroscopic pattern has been described in patients with PBC and Raynaud's phenomenon (RP), possibility for anticentromere antibodies (ACA) and overlap with another systemic autoimmune disease, the most frequent being Systemic Sclerosis (SSc) (association with a prevalence up to $17 \%$ of PBC patients called Reynolds Syndrome) (2). However, studies are scarce and not very detailed in terms of the differential capillaroscopic findings between patients with PBC with/without SSc and with/without RP.

Objectives: To analyze the differences between clinical, serologycal and capillary morphological alterations observed in three different groups of patients with PBC: patients with PBC alone (PBC-A), patients with PBC and RP (PBC-RP) and patients with Reynolds Syndrome (PBC-RS).

Methods: Periungual capillaroscopy was performed on 12 patients with PBC-A, 10 with CBP-RP and 13 with PBC-RS, who received follow-up in our systemic diseases and hepatology monographic outpatients. Capillaroscopy was made with USB Digital Microscope Dino-Lite ${ }^{\circledR}$ epiluminiscence video. The capillaroscopic alterations were according to a semiquantitative method. All patients were given a detailed clinical evaluation. Variables related to clinical, serological and capillaroscopic parameters were collected. A comparative study was done.

Results: Of the 36 patients analyzed, 32 (88.9\%) were women, with no sex differences between the three groups. The median age at PBC diagnosis was $50+-12.8$ years in PBC-A group, $60.5+-15$ years in PBC$\mathrm{RP}$ and 62 years in PBC-RS, showing significant difference between the first group and the other two ( $p$ 0.039). 14 patients had other systemic diseases: 1 hemolytic anemia, 1 SLE, 2 Psoriasis, 1 PTI and 9 Sicca. The only clinical parameter with significant difference between the three groups was association with Sicca: 3 (25\%) PBC-A, 5 (50\%) PBC-RP and $12(85.7 \%)$ PBC-RS, p 0.002 . Twenty five (73.5\%) patients had positive AMA, with no differences between groups. All 11 patients who had ACA were from the PBC-RS group. Statistically significant differences observed between the capillaroscopy parameters were the presence of capillary dilatations $[5$ (41.7\%) $\quad$ PBC-A, 5 (50\%) PBC-RP, 11 (84.6\%) PBC-RS, p 0.03] and pathological hemorrhages [1 (8.3\%) in PBC-A, 2 $(22.2 \%)$ PBC-RP, 11 (78.6\%) PBC-RS, p <0.001] as well as the presence of a different capillaroscopic pattern ( $p<0.001)$ : normal or nonspecific in $9(75 \%)$ of PBC-A, 4 (44.4\%) PBC-RP and 2 (15.4\%) PBC-RS; connective tissue disease suggestive pattern in $3(25 \%)$ of PBC-A, 5 $(55.6 \%)$ of PBC-RP and $1(7.7 \%)$ of PBC-RS; sclerodermiform pattern in $10(76.9 \%)$ PBC-RS, and none of the other two groups. We did not find significant differences in the presence of simple tortuosities, complex tortuosities, branched capillaries or capillary loss. No capillaroscopic parameters correlated with other systemic diseases.

Conclusion: This preliminary study shows an evolutionary trend in some clinical (age at PBC diagnosis, sicca asocciation) and capillaroscopic parameters (capillary dilatation, hemorrhages, general capillaroscopic pattern) in patients with PBC-A, PBC-RP and PBC-RS, which may suggest three different phenotypic expressions of the same pathogenic process.

\section{REFERENCES:}

[1] Fonollosa V, et al. Morphologic capillary changes and manifestations of connective tissue diseases in patients with primary biliary cirrhosis. Lupus. 2001;10(9):628-31.

2. Zheng B, et al. Prevalence of Systemic Sclerosis in Primary Biliary Cholangitis Using the New ACR/EULAR Classification Criteria.
Disclosure of Interests: None declared

DOI: 10.1136/annrheumdis-2019-eular.3538

\section{THU0609 ULTRASOUND AS A USEFUL TOOL IN THE DIAGNOSIS OF RHEUMATOID ARTHRITIS IN PATIENTS WITH UNDIFFERENTIATED ARTHRITIS}

Marwin Gutierrez ${ }^{1}$, Chiara Bertolazzi ${ }^{1}$, Edwin Castillo², Denise Clavijo Cornejo ${ }^{1}$, Luis Carlos Rodriguez Delgado ${ }^{1}$, Jaime Mendoza Torres ${ }^{1}$, Carlos Pineda ${ }^{3}$, Pedro Santos-Moreno $0^{4} .{ }^{1}$ Instituto Nacional de Rehabilitación Luis Guillermo Ibarra Ibarra, Division of Musculoskeletal and Rheumatic Diseases, Tlalpan, Mexico; ${ }^{2}$ Center of Rheumatoid Arthritis BIOMAB, Rheumatology, Bogota, Colombia;

${ }^{3}$ Instituto Nacional de Rehabilitación Luis Guillermo Ibarra Ibarra, Division of Musculoskeletal and Rheumatic Diseases, Mexico City, Mexico; ${ }^{4}$ Center of Rheumatoid Arthritis BIOMAB, Bogota, Colombia

Background: Nowadays, rheumatologists face challenges in finding an effective method to classify and treat patients with undifferentiated arthritis (UA). There is a need for new tools that could ensure accurate characterization of inflammatory processes in these patients.

Objectives: To investigate if a characterization of UA patients using US may help to fulfill the 2010 ACR/EULAR RA classification criteria in a real-life cohort.

Methods: We conducted a cross sectional study in two rheumatology care clinics. Patients not fulfilling the 2010 ACR/EULAR RA criteria were included. On the examination day, all patients underwent a physical examination, radiographs and US. The 7-joint US score (US 7) was adopted to scan all patients. US was performed according to EULAR criteria and interpreted by OMERACT definitions. Greyscale and power Doppler synovitis and tenosynovitis were scored. Bone erosions were also evaluated during the US examination.

Results: A total of 204 patients were included. The diagnosis was modified from UA to RA in $86(42.1 \%)$ patients. The greater proportion of synovitis detected by US was the main parameter that allowed changing the diagnosis from UA to RA, and modified the final score of the 2010 ACR/EULAR classification criteria, from a mean $( \pm S D)$ of $4.6(0.5)$, by clinical examination, to $6.5(0.6)$ by US. The changes in the score of the 2010 ACR/EULAR classification criteria were from score 4 to score 6 in $6(7 \%)$ patients; from 4 to 7 in $24(27.9 \%)$ patients; from 5 to 6 in 42 $(48.8 \%)$ patients; from 5 to 7 in $5(5.8 \%)$ patients and from 5 to 8 in 5 $(5.8 \%)$ patients.

In addition to synovitis, a wide range of tenosynovitis and bone erosions were detected by US. Synovitis was more frequently detected in $2{ }^{\text {nd }} M C P$ followed by $2^{\text {nd }} M T P j$ and $5^{\text {th }} M T P j$. The tendons of the wrist, $2^{\text {nd }}$ and $3^{\text {th }}$ finger were the most affected. In relation to bone erosions, $2^{\text {nd }} \mathrm{MCPj}$ and $5^{\text {th }}$ MTPj where the joints with more proportion of anatomical damage.

Conclusion: US demonstrated to be useful to help accurately classify as RA, patients previously diagnosed with UA.

Disclosure of Interests: Marwin Gutierrez: None declared, Chiara Berto lazzi: None declared, Edwin Castillo: None declared, Denise Clavijo Cornejo: None declared, Luis Carlos Rodriguez Delgado: None declared, Jaime Mendoza Torres: None declared, Carlos Pineda: None declared, Pedro Santos-Moreno Grant/research support from: Dr Santos has received research grants from Janssen, Abbvie and UCB, Speakers bureau: Dr Santos has received speaker fees from Sanofi, Lilly, Bristol, Pfizer, Abbvie, Janssen and UCB

DOI: 10.1136/annrheumdis-2019-eular.6115

\section{THU0610 \\ ANALYSIS OF ANTINUCLEAR ANTIBODY ANTIBODIES POSITIVITY AND THEIR MAJOR KARYOTYPES IN PATIENTS WITH AUTOIMMUNE DISEASES AND HEALTHY SUBJECTS}

Qiujing Wei, Yutong Jiang, Jiewen Xie, Jun Qi, Jieruo Gu. the Third Affiliated Hospital of Sun Yat-sen University, Guangzhou, China

Background: Autoantibodies, especially antinuclear antibodies (ANA), play an important role in the diagnosis and differential diagnosis of autoimmune disease (AID), disease monitoring, efficacy observation and pathogenesis research.

Objectives: Our aim was to investigate the rate of ANA positivity and their major karyotypes in different AIDs and healthy controls.

Methods: The distribution and positive rate of Antinuclear antibody (ANA) karyotypes detected by indirect immunofluorescence in 3704 patients with AID and 1073 healthy subjects were retrospectively analyzed.

Results: The positive rate of ANA in different AID groups was $90.7 \%$ $(1845 / 2034)$ in SLE, $54 \%(525 / 973)$ in $\mathrm{RA}, 86.4 \%(267 / 309)$ in SS 